\title{
Computational Aspects of Sustainability
}

\author{
George E. Halkos ${ }^{1}\left[\mathbb{D} \cdot\right.$ Kyriaki D. Tsilika ${ }^{1}[$
}

Published online: 20 June 2021

(c) The Author(s), under exclusive licence to Springer Science+Business Media, LLC, part of Springer Nature 2021

\section{Introductory Overview}

The main purpose of the special issue is to feature the computational practice of green policy performance measurement. Computing the progress of green economy includes topics as indicators and measures to characterize environmental sustainability, methodological issues to indicate and present spatiotemporal patterns of resource use and pollution, computational frameworks for comparisons of environmental management among economies/economic sectors/socio-economic systems, computational techniques to define the structure, dynamics and change in ecosystems. Results are discussed in support of green policies.

The applied theoretical and analytical contributions guide policy-makers and government officials in designing new policy scenarios for the investigation of sustainability and the appropriate advanced and updated methods to be used. The empirical contributions provide evidence to support and inform current policy debates.

Collected here are some empirical and theoretical developments for the development and application of environmental models. The special issue aims to exchange information, knowledge, and ideas through research contributions from different fields-economics, computer sciences, and analytics.

This special issue is directed specifically at the Computational Economics audience and is intended to provide researchers with sufficient background to investigate the multiple dimensions of sustainable development and to introduce common or innovative tools and approaches to analyze and understand them with the audience assumed to have some basic knowledge of environmental modeling and computational integration of statistical techniques.

George E. Halkos

halkos@uth.gr

1 Laboratory of Operations Research, Department of Economics, University of Thessaly, 78, 28hs

Octovriou St, 38333 Volos, Greece 


\section{Main Characteristics of the Existing and Current Research on Sustainable Development}

Sustainable development is a multidimensional concept. Interconnectedness between segments of the entire system called sustainable development is undoubted. In the last five years there is significant research interest in computational tackling of the various environmental problems and environmental policy implications.

Research activity on green policy performance measurement is present in several disciplines such as computer science, finance, economics, financial engineering, physics, engineering, social sciences, environmental science, energy, business, management and accounting, mathematics, decision sciences. Relevant documents were identified from selected research databases, namely Scopus, Google Scholar, and EBSCOhost, by executing search queries for keywords like, 'computing', 'SDGs', 'sustainability', and 'green policy'. The results are reported in Table 1.

This special issue collected papers presenting new contributions to this literature with a special focus on manuscripts adopting computable models for green growth, analyzing policy interactions with quantitative methods, performing cost/benefit analysis based on economic-ecological transformations.

Understanding sustainable development linkages and achieving the required policy trade-offs across the environmental, economic, and social spheres requires a wide range of tools and methodologies.

This special issue aims to consider the "economic growth and environment" dispute in terms of the most updated and efficient methods applied in various modeling techniques together with the use of better and larger data sets. Above all, the computational tools and methodologies for studying sustainable development in all its dimensions: environment, health, economic impacts.

The papers included developing core theories, frameworks, and features that underpin research in sustainability issues (see Fig. 1). Coverage areas include:

Table 1 Review of the literature on computational tools and methodologies for studying sustainability

The literature search results

\begin{tabular}{|c|c|c|}
\hline $\begin{array}{l}\text { Scientific publish- } \\
\text { ing knowledge } \\
\text { base }\end{array}$ & Keywords & Search results \\
\hline \multirow[t]{3}{*}{ Scopus } & Computing AND sustainability & 3130 documents \\
\hline & $\begin{array}{l}\text { "Sustainable development goals" AND comput- } \\
\text { ing }\end{array}$ & 527 documents \\
\hline & $\begin{array}{l}\text { Computing AND sustainability AND "green } \\
\text { policy" }\end{array}$ & 88 documents \\
\hline Google scholar & $\begin{array}{l}\text { Computing AND sustainability AND "green } \\
\text { policy" }\end{array}$ & 511 results \\
\hline \multirow[t]{2}{*}{ EBSCOhost } & $\begin{array}{l}\text { Computing AND sustainability AND "green } \\
\text { policy" }\end{array}$ & 35,724 results (years 2016-2020) \\
\hline & $\begin{array}{l}\text { "Sustainable development goals" AND comput- } \\
\text { ing }\end{array}$ & 88 results (years 2016-2020) \\
\hline
\end{tabular}


Fig. 1 Key themes and topics of "computational aspects of sustainability" published by computational economics (Springer)

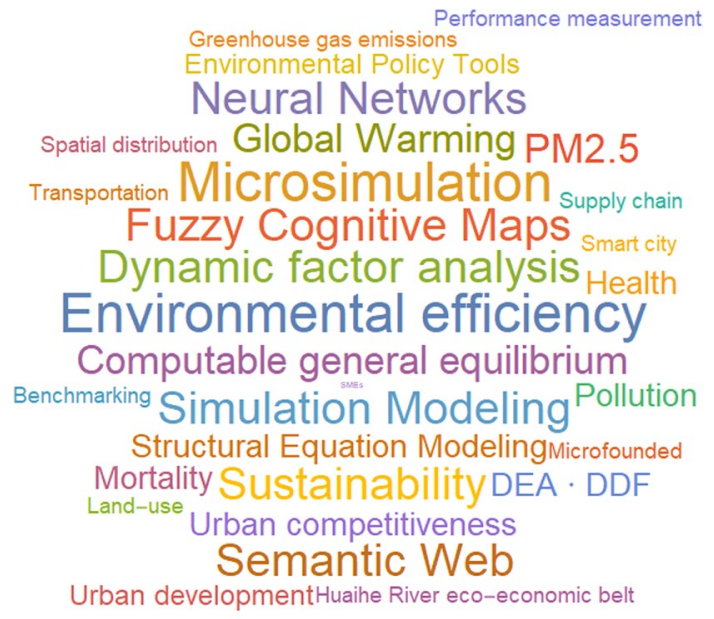

Greenhouse gas emissions

Environmental Policy Tools

Neural Networks

Spatial distribution Global Warming PM2.5

Microsimulation ${ }_{\text {supply chain }}$

Fuzzy Cognitive Maps

Dynamic factor analysis Health

Environmental efficiency

Computable general equilibrium

Structural Equation Modelingmicrofounded

Mortality Sustainability DEA · DDF

Semantic Web

Semantic Web

Urban developmentHuaihe River eco-economic belt

- Causal relationships that exist in the field of Global Warming

- Dynamic interactions between the economy, energy, climate change

- Predictions of Global Warming Impacts

- Trade-offs between environmental and economic performance

- Effects of urban planning and transportation policies,

- Urban land use, mobility patterns, and their environmental impacts

- Quantitative measurement and evaluation of urban competitiveness

- SMEs' supply chain sustainability performance/sustainability performance measurements of SMEs

- Pollution and health

- Environmental efficiency

\section{Editors' Overview of Research Contributions}

The authors of the selected papers seek and suggest the computational framework to analyze the environmental dimensions of the sustainable development. Specifically:

Song and Xie (2021) measure and compare regional urban competitiveness in the eco-economic context. They establish an evaluation model with the help of the dynamic factor analysis method which aims to quantitatively measure the degree and ranking of the competitiveness of cities. The model is tailored to reflect changes in the competitiveness of cities in terms of time, but also to compare the competitiveness of different cities in the same period. The computational approach uses STATA software and appropriate panel data according to the basic ideas of dynamic factor analysis. Huaihe River eco-economic belt construction is considered by the authors as a typical case study in which the measurement approach of urban competitiveness can be applied and attain policy integration. The paper concludes that understanding the different developmental strengths and comprehensive competitiveness of each city is key to successful regional ecological economy construction. 
On the path of "eco-friendly policies through computable models" a new approach to modeling can be found in (Tikoudis \& Oueslati, 2021) with an urban Computable General Equilibrium model, the Multi-Objective Local Environmental Simulator (MOLES). The model aims to assess the performance of local and national policy instruments that target transportation, energy consumption from mobile sources, and land use in urban areas. It is also designed to capture the synergetic effects of urban planning and transportation policies. The research results provide theoretical reference for the evaluation of the environmental and economic impacts of urban policies using the urban area of Auckland, New Zealand as the sample. The version of MOLES for Auckland is considered by the authors as a laboratory in which landuse and transportation reforms for similar cities can be examined, yielding policy recommendations with a considerable degree of external validity. The model helps policy makers distinguish potential best practices, i.e. policy interventions that are economically efficient, environmentally effective, and balanced from a fiscal and distributional viewpoint. MOLES is also a platform to detect worst practices.

Indicating and linking the environmental dimensions of the sustainable development, Tsadiras et al. (2021) develop a model of the causal relationships that exist in the field of Global Warming. A Semantic Web-based software tool/application called "Web-FCM" is designed and implemented using the well-established Artificial Intelligence technique of Fuzzy Cognitive Maps (FCMs). The model visually simulates the FCM dynamic behavior and studies the equilibrium that the FCM dynamic system reaches. Common frontend and backend web development technologies such as HTML, JavaScript, CSS and Java Server Pages (JSP) were chosen for the implementation of the proposed Web-FCM system. The most important component of the software is the use of Semantic Web technologies to represent the related entities (concepts, scenarios etc.) and manipulate data. Policy makers can use this technique and tool to make predictions by viewing dynamically the consequences that the system predicts to their imposed scenarios and share them through the world wide web. An FCM model that concerns Global Warming demonstrates the capabilities of the proposed Web-FCM system.

A new framework for measuring and managing the supply chain sustainability for small and medium-sized enterprises is proposed by Dey et al. (2021). They have managed to identify empirically the conditions that govern the economic, operational, social, and environmental practices and the performance of the involved SMEs and propose a package of amelioration measures to be examined through benchmarking against sectoral best practice. The proposed model exploits the advantages of a joint SEM and DEA analysis: SEM and DEA analysis has offered the ability to extract through SEM suitable overall input and output latent constructs of sustainability practices and performances. This approach is more powerful than similar research that used separately DEA and SEM for sustainability analysis. This suggested joint approach was empirically validated in two varied set-ups: Midlands in the United Kingdom and Normandy in France.

Effects of social variables on the environment is a key dimension of sustainable development. Halkos and Argyropoulou (2021) examine the effects of air pollution on health, using the nonparametric approach of DEA using MaxDEA Pro. They employed the statistical procedure of bootstrapping, implemented with 
bootstrapping bias correction. Two DEA models are specified with capital and labor as inputs and GDP/c as desirable and mortality from exposure to $\mathrm{PM}_{2.5}$ as undesirable (bad) outputs in the first and environmentally related tax revenue as additional input in the second specification. The main result is the ranking of a set of 18 European countries for the years 2000, 2005, 2010, 2014, 2015 and 2016, according to their assigned efficiency scores. Differences in efficiencies extracted may be due to the different chemical composition of the particles stemming from different sources of emissions with varying chemical characteristics.

The selected papers are fully consistent mainly with five sustainable development goals, namely, goal 3: Good Health and Well Being, goal 7: Affordable and Clean Energy, goal 11: Sustainable Cities and Communities, goal 12: Responsible Consumption and Production, goal 13: Climate Action.

\section{References}

Dey, P. K., Yang, Gl., Malesios, C., et al. (2021). Performance management of supply chain sustainability in small and medium-sized enterprises using a combined structural equation modelling and data envelopment analysis. Computational Economics. https://doi.org/10.1007/s10614-019-09948-1

Halkos, G., \& Argyropoulou, G. (2021). Pollution and health effects: A nonparametric approach. Computational Economics. https://doi.org/10.1007/s10614-019-09963-2

Song, M., \& Xie, Q. (2021). Evaluation of urban competitiveness of the Huaihe River eco-economic belt based on dynamic factor analysis. Computational Economics. https://doi.org/10.1007/ s10614-019-09952-5

Tikoudis, I., \& Oueslati, W. (2021). MOLES: A new approach to modeling the environmental and economic impacts of urban policies. Computational Economics. https://doi.org/10.1007/ s10614-019-09962-3

Tsadiras, A., Pempetzoglou, M., \& Viktoratos, I. (2021). Making predictions of global warming impacts using a semantic web tool that simulates fuzzy cognitive maps. Computational Economics. https:// doi.org/10.1007/s10614-020-10025-1

Publisher's Note Springer Nature remains neutral with regard to jurisdictional claims in published maps and institutional affiliations. 